\title{
Extended Selected Abstracts from Workshop of the Geriatric section of Hellenic Physical Medicine and Rehabilitation Society
}

\author{
9 June 2018, \\ KAT Hospital, Kifissia, Greece
}

All published work is licensed under Creative Common License CC BY-NC-SA 4.O (Attribution-NonCommercial-ShareAlike)

\section{INTRODUCTION IN FRAILTY \\ Yannis Dionyssiotis}

One way to understand frailty is by comparison with the traditional concept of disease. Fifty years ago, the typical patient admitted to the hospital was suffered from a disease and was treated based on clinical diagnosis. Nowadays, most of the patients are suffering from multiple diseases and the target to therapy is shifted to regain the functionality of the patients instead to treat a disease only ${ }^{1}$.

Frailty is important because is associated with many serious conditions. Hospitalisation, falls, disabilities and death relate to the presence of frailty².

Ageing may be conceptualised as a process of progressively increased entropy coupled with reduction and subsequently failure of the homeostatic mechanisms. Homeostatic equilibrium is maintained by robust function and interconnections between multiple physiologic systems. When one system is dysregulated or impaired, as in a specific disease, disability can result in the specific areas of function affected by the disease. In younger subjects the disease usually does not result in disability. There is a threshold to be overtaken in order to be disabled which is not reached in this case. However, later and during aging the derangement of general homeostatic mechanisms characteristic of frailty induces a multiple systems impairment which passes the disability threshold because of development of multiple diseases and leads to disability ${ }^{3}$.

There is general agreement that frailty results from underling physiologic and/or biologic alterations that are age-associated and maybe compounded by single or multiple diseases, or even be an end-stage outcome of several disease. Key systems thought to be involved in the development of frailty include muscoloskeletal, hormonal, immune and inflammatory systems with likely contributions from the autonomic anc CNS. Sarcopenia or loss of muscle mass if thought to $b$ a central manifestation of frailty ${ }^{3}$.

The pathway of frailty starts with modification of physiology or biological alterations associated with ageing which may be deteriorated from diseases or even could be the final stage of diseases.

Basic systems which is believed to interfere with frailty include musculoskeletal, hormones, immune function, inflammation and may be contributed from autonomic nervous system.

Frailty is defined as a multi-system impairment associated with increased vulnerability to stressors and describes individuals who are at increased risk of adverse health outcomes (disability, admissions in hospitals, institutionalization, mortality).

Frailty includes as basic components: disease, sarcopenia, reduced resting metabolic rate, total energy expenditure, undernutrition, reduced strength and power, reduced oxygen capacity. These components are dysregulated energetics as well as altered physiologic functioning and create a vicious cycle 4 .

The comprehensive geriatric assessment (CGA), may be an option for characterizing frailty. However, due to its length is difficult to be used and requires geriatric training to carry it out and more evidence that is proper to identify subjects as frail. So, it would be of great importance to include a specific measure of frailty, in the CGA.

Fried et al described frailty as a medical syndrome (assessed as the Fried frailty phenotype). Frailty criteria (which apply to the physical frailty) include shrinking, weakness, poor endurance and energy, slowness, reduced physical activity or exercise. All these criteria correspond to the following characteristics weight loss, low grip strength, poor endurance and energy, self-reported exhaustion, walking speed/Kcal expended per week, level of physical activity, respectively. Three or more of the above criteria define a subject as a frail. The Fried physical frailty phenotype might capture sarcopenic older adults as the criteria are directly related to the development of sarcopenia. Individuals can be categorized as non-frail (O Fried criteria present), pre-frail or intermediate (1-2 criteria) or frail ( $\geq 3$ criteria) ${ }^{5}$.

Rockwood and Mitnitski describe it as an accumulation 
of deficits (assessed as the Rockwood frailty index). The Rockwood Index can be adapted in a similar manner to provide a proportionate expression of the number of deficits. This type of model is based on the calculation of indices, obtained with a scoring system covering several multidimensional domains that are associated with increases in the risk of functional decline and/or adverse events ${ }^{6}$.

The 5-item FRAIL scale was used for screening for frailty. There are 5 components: fatigue, resistance, ambulation, illnesses, and loss of weight. Frailty scores range from 0-5 (i.e., 1 point for each component; $0=$ best to $5=$ worst) and represent frail (3-5), pre-frail ( $1-2)$, and robust (0) health status ${ }^{7}$.

The mini nutritional assessment questionnaire (MNA) is a very common tool we are using in a clinical practice. It would be useful in frailty screening as it includes questions about weight loss, mobility, depression and dementia ${ }^{8}$. Treating undernutrition is very important in frailty. Malnutrition is a high-risk factor for frailty, therefore we need to identify at-risk population early. MNA ${ }^{\circledR}$-SF (Mini Nutritional Assessment ${ }^{\circledR}$ short-form) is one of the best validated screening tools to identify malnourished or at risk of malnutrition older adults and has been known to predict frailty both in community dwelling older adults and hospital patients ${ }^{9,10}$.

Gait speed is a prognostic value in well-functioning of older people. Decreased gate speed below $1 \mathrm{~m} / \mathrm{sec}$ was associated with death, hospitalisation and persistent severe lower extremity limitation. The rate of events per participant/year were increased in subjects with gait velocity less than $1 \mathrm{~m} /$ sec in the 6 minutes test ${ }^{6}$.

When we are trying to assess frailty in community dwelling elderly subjects, we can use the Gérontopôle Frailty Screening Tool (GFST). The GFST instrument involves administration of the Basic Activities of Daily Living (ADL) scale. A score lower than $5 / 6$ on this scale identifies individuals with relevant dysfunction in basic activities of daily life. Finally, the general practitioner is invited to express their opinion on the frailty status of the individual. The clinical judgment of the general practitioner is used to determine whether, after the evaluation of the criteria, they believe the person is frail or not ${ }^{11}$.

Each intervention in frailty is the purpose to tackle specific affected outcomes. For example, interventions with exercising physical activity, target mobility problems, depression issues or even inflammation. Recently the LIFE-P study published effects of physical activity intervention on measures of physical performance ${ }^{12}$.

Another entity beyond physical frailty is cognitive frailty which is a heterogeneous clinical manifestation characterised by the presence of physical frailty and cognitive impairment with exclusion of concurrent Alzheimer disease dementia or other dementias. Cognitive frailty may represent a precursor of neurogenerative processes. A potential for reversibility may also characterise this entity and a psychological component of the condition must be taken into consideration. What is important with cognitive frailty is the reversibility before the patient passes to the definitive frailty condition ${ }^{13}$.

According to Prof. Vellas B., multi-domain interventions are superior compared to single intervention techniques. This is the case both in the treatment of physical and cognitive frailty. Nutritional supplements, physical and cognitive exercises, pharmacological supplementation as well as public awareness education programmes are common intervention as both entities are sharing the same pathogenetic pathways ${ }^{8}$.

To conclude: Frailty is characterized by an impaired ability to cope with challenges in health and reduced ability to regain a stable health status, possibly related to reduced functional reserve. Frail older persons are at risk for multiple adverse health outcomes. No standard definition of frailty is available. Frail older persons require intensive and multidimensional continuous care.

\section{References}

1. Rodriguez-Manas L, Fiart C, Mann G, et al. Searching for an operational definition of frailty: a Delphi method based consensus statement: the frailty operative definition-consensus conference project. J Gerontol A Biol Sci Med Sci 2013;68:62-67

2. Clegg A, Young J, lliff e S, Rikkert MO, Rockwood K. Frailty in elderly people. Lancet 2013;381:752-62

3. Hazzard's Geriatric Medicine and Gerontology, $6^{\circ}$ th Edition.

4. Fried LP, Tangen CM, Walston J, et al. Frailty in older adults: evidence for a phenotype. J Gerontol A Biol Sci Med Sci 2001 ;56:M1 46-56.

5. Rockwood K, Song X, Macknight C, Bergman H, Hogan DB, McDowell I, Mitnitski A.A global clinical measure of fitness and frailty in elderly people. CMAJ 2005; 173(5):489-95

6. Cesari M, Kritchevsky SB, Penninx BW, Nicklas BJ, Simonsick EM, Newman AB, Tylavsky FA, Brach JS, Satterfield S, Bauer DC, Visser M, Rubin SM, Harris TB, Pahor M. Prognostic value of usual gait speed in well-functioning older people - results from the Health, Aging and Body Composition Study. J Am Geriatr Soc 2005;53(10): 1675-80.

7. Morley JE, Malmstrom TK, Miller DK. A simple frailty questionnaire (FRAlL) predicts outcomes in middle aged African Americans. J Nutr Health Aging 2012;16(7):601-8.

8. Dionyssiotis Y, Chhetri JK, Piotrowicz K, Gueye T, Sánchez E. Impact of nutrition for rehabilitation of older patients: report on the 1st EICA ESPRM - EUGMS train the trainers course. Eur Geriatr Med 2017; 8:183-90

9. Jürschik P, Botigué T, Nuin C, Lavedán A. [Association between Mini Nutritional Assessment and the Fried frailty index in older people living in the community]. Med Clin (Barc) 2014;143(5): 191-5.

10. Dorner TE, Luger E, Tschinderle J et al Association between nutritional status (MNA ${ }^{\circledR}-\mathrm{SF}$ ) and frailty (SHARE-FI) in acute hospitalised elderly patients J Nutr Health Aging 20 14; 18(3):264-9

11. Vellas B, Balardy L, Gillette-Guyonnet S, Abellan Van Kan G, GhisolfiMarque A, Subra J, Bismuth S, Oustric S, Cesari M. Looking for frailty in community-dwelling older persons: the Gérontopôle Frailty Screening Tool (GFST). J Nutr Health Aging 20 13; 17(7):629-31.

12. LIFE Study Investigators, Pahor M, Blair SN, Espeland M, Fielding R, Gill TM, Guralnik JM, Hadley EC, King AC, Kritchevsky SB, Maraldi C, Miller ME, Newman AB, Rejeski WJ, Romashkan S, Studenski S. Effects of a physical activity intervention on measures of physical performance: Results of the lifestyle interventions and independence for Elders Pilot (LIFE-P) study. J Gerontol A Biol Sci Med Sci. 2006 
Nov;61(11):1157-65. Erratum in: J Gerontol A Biol Sci Med Sci 2007;62(3):337

13. Kelaiditi E, Cesari M, Canevelli M, van Kan GA, Ousset PJ, GilletteGuyonnet S, Ritz P, Duveau F, Soto ME, Provencher V, Nourhashemi F, Salvà A, Robert P, Andrieu S, Rolland Y, Touchon J, Fitten JL, Vellas B; IANA/IAGG. Cognitive frailty: rational and definition from an (I.A.N.A./I.A.G.G.) international consensus group. J Nutr Health Aging 2013;17(9):726-34

\section{URINARY INCONTINENCE IN ELDERLY}

\section{Angeliki Galata}

Urinary incontinence (UI) is defined as any kind of involuntary urination, irrespective of the volume ${ }^{1}$. It is an important medical and socioeconomic issue, especially with aging. Moderate to severe UI affects $7 \%$ of women 20 to 39 years of age, $17 \% 40$ to 59 years of age, $23 \%$ 60 to 79 years of age, and $32 \% \geq 80$ years of age ${ }^{2}$. The prevalence in men is approximately one third that of women, until it equalizes in the ninth decade and the rate of UI reaches $60-70 \%$ in the elderly in nursing homes. Given the worldwide demographic trends, this medical condition is expected to increase even further.

Seniors suffering from UI carry a profound psychological burden, causing a negative impact on quality of their life, including self-concept and self-esteem, depression and social isolation. They show significant morbidity (due to falls and fractures, skin complications), increased hospital admissions, as well as great functional impairment. UI represents a negative prognostic factor for other comorbidities, such as cerebrovascular diseases ${ }^{3,4}$, while there is an increased likelihood of institutionalization ${ }^{5}$ or even increased mortality ${ }^{6}$. The consequences of incontinence extend beyond the affected individual to their caregivers, leading to physical and emotional stress, as well as heavy financial burden ${ }^{7,8}$.

In older persons, UI represents a geriatric syndrome with several risk factors that include age-related changes in physiology (i.e., deterioration of diurnal rhythm of antidiuretic hormone, changes in bladder's and pelvic floor's function), comorbidities (i.e., depression, transient ischemic attacks, stroke, dementia, congestive heart failure, fecal incontinence, constipation), medications (i.e., opioid analgesics, diuretics), obesity and especially functional impairments (i.e., vision's deterioration, motor limitation, cognitive disorders) ${ }^{9-11}$. Apart from personal risk factors, many environmental causes can contribute to UI, such as non-accessible toilets or lack of assistance from a caregiver.

Urinary incontinence is categorized according to pathophysiology and clinical presentation into three main categories ${ }^{1}$ : i) stress urinary incontinence (SUI), ii) urge urinary incontinence (UUI), and iii) mixed urinary incontinence (MUI).

i) SUI is defined as an involuntary loss of urine during activities with increasing intra-abdominal pressure (i.e. coughing, laughing, exercise). Among elderly, SUI shows a decreasing trend with aging and it occurs due to impaired urethral sphincter support or/and due to damage impairing urethral closure, as may occur from urologic surgery (i.e., radical prostatectomy) and neurological lesions (i.e., spinal cord injury). Other risk factors include menopause, obesity, constipation, and smoking.

ii) UUI increases in frequency with aging and is defined as the leakage that is associated with urgency. It is usually associated with detrusor overactivity (DO). However, up to $40 \%$ of continent healthy older adults have DO on urodynamic testing ${ }^{12}$, suggesting that UUI requires the co-existence of other etiologic factors as well, such as age-related reasons, bladder outlet obstruction, local bladder irritation (i.e., infection, stones, tumor), lesions in central nervous system, or idiopathic.

iii) MUI is the involuntary loss of urine that is presented with overlapping features of both urge and stress UI (one of them in predominance).

Moreover, UI might be associated with impaired bladder emptying and an elevated postvoid residual (PVR). It occurs either due to bladder's outlet obstruction (i.e., prostate hypertrophy, large cystocele), or due to disorder of detrusor's contractility, or both ${ }^{13}$. On the one hand, acontractile or hypocontractile detrusor because of peripheral neuropathy (i.e., diabetes, $B_{12}$ deficiency), sacral cord and roots' lesion (i.e., spinal stenosis, tumour) can cause incomplete micturition. On the other hand, under specific circumstances also detrusor contractility may not be sufficient and provoke increased PVR (detrusor hyperactivity with impaired contractility- DHIC) ${ }^{12}$.

In addition, leakage in seniors can be caused or worsened by a variety of interacting risk factors, including comorbidities, medication, age-related physiological changes and environmental barriers causing functional limitations. This condition is frequently named as "transient" UI, because of the potential reversibility of the contributing factors mentioned, but if they are not managed, it will be a chronic and often progressive condition.

Consequently, when dealing with UI in elderly, it is important to use a holistic way of approaching and try to seek for all the possible risk factors whose amelioration may improve symptoms, as well as patients and their caregivers' quality of life ${ }^{14}$. Evaluation must be thorough and multifactorial. The very first step is to identify the individuals in need, because one-half of them may not report their symptoms due to their embarrassment or their perception that $\mathrm{UI}$ is a natural consequence of aging ${ }^{15}$. On the other hand, UI may not be well addressed by the clinicians, due to their reluctance to face this issue.

According to International Continence Society, the evaluation of UI in older population should include a detailed history in order to obtain all the useful information about symptoms of lower urinary system (incontinence, urgency, 
nocturia, changes of urine flow) and their characteristics (onset, frequency, severity). All the possible associated risk factors should be documented and especially those potentially reversible conditions that can be easily remembered by the mnemonic DIPPERS [Delirium, Infection (urinary tract), Pharmaceuticals, Psychological, Excess fluid (in/out), Restricted mobility, and Stool impaction (and constipation) $]^{16}$. The burden of patients and their caregivers should be assessed (even by specific questionnaires), as well as their expectations from the therapeutic procedure. Since the commitment to recommendations is crucial for the overall outcome, we should be able to recognize those patients who seem to be unwilling to be adherent to them.

A comprehensive physical examination should include a cognitive and functional evaluation (in order to diagnose motor limitations, need for assistance in transfers/ ambulation), a neurological examination (sacral cord integrity- perineal sensation, anal sphincter tone/contraction, anal reflexes and bulbocavernosus reflex), a rectal examination for potential stool impaction and prostate enlargement in men, a vaginal examination for checking vaginal mucosa in women and pelvic floor assessment for organ prolapse ${ }^{10}$. If SUI is possible, cough test should be performed ${ }^{17}$.

The diagnostic evaluation to be considered complete should also include some basic diagnostic tests (urine analysis, blood test for kidney function) and a three-days bladder diary that reports fluid intake, micturition, episodes of incontinence, accompanied by weighting of the used pads.

In cases of failed conclusive assessment or ineffective therapeutic treatment, more extensive diagnostic tests should be considered, such as ultrasonography of the urinary tract (with measurement of PVR), uroflowmetry, urine culture (if infection is suspected), complete gynaecological examination, urodynamics (if surgical procedure is planned), and urethrocystoscopy (if more severe symptoms are present i.e., blood, unexplained pelvic pain).

Initial management should be individualized (taking into account patients' mobility, motivation, and cognitive competence) and stepped, starting with noninvasive conservative treatment and then adding medications, and finally minimally invasive or more extensive surgery, if necessary ${ }^{18}$.

Conservative and behavioural management include modification of lifestyle (i.e., weight loss, smoking cessation, control of fluid intake), bladder training (habit retraining, prompted voiding) and pelvic floor muscles exercises with biofeedback and/ or electrotherapy, when applicable. For strengthening of pelvic floor, magnetic stimulation can also be used in elderly with uncomplicated mild SUI.

Any drug treatment (i.e., antimuscarinics) should be started with a low dose and titrated with regular monitoring due to potential risk of side effects (i.e., cognitive deterioration, constipation, dry mouth). Occasionally, a combination of the methods above should be considered for better outcomes. Minimally invasive methods, such as sacral nerve stimulation, and surgical management are recommended when conservative and drug treatment have failed in individuals with more complicated and severe symptomatology and if their general condition permits such interventions.

$\mathrm{UI}$ is one of the common medical conditions seen in elderly causing a heavy economic, psychological and functional impact on patients and their social environment. Concerning the aging of the population, this issue will become even larger for the health care systems and society. However, most of the time individuals avoid mentioning their symptoms and do not seek for medical treatment. So it is essential to identify the older people who suffer from UI and after a holistic evaluation to recognize potential reversible causes and recommend the appropriate management. Conservative treatment is proposed as the first therapeutic choice. Behavioral methods can be combined with pharmachological agents for better results but with great respect to cost, adverse effect profile, patient comorbidities, and administration. In cases of ineffectiveness of that approach, more invasive interventions should be considered.

\section{References}

1. Abrams P, Cardozo L, Fall M, et al. The standardisation of terminology of lower urinary tract function: report from the Standardisation Subcommittee of the International Continence Society. Neurourology and urodynamics 2002;21(2):167-178.

2. Nygaard I, Barber MD, Burgio KL, et al. Prevalence of symptomatic pelvic floor disorders in US women. Jama 2008;300(11):13111316.

3. Baztan JJ, Arias E, Gonzalez N, Rodriguez de Prada MI. New-onset urinary incontinence and rehabilitation outcomes in frail older patients. Age and ageing 2005;34(2): 172-175.

4. Patel M, Coshall C, Rudd AG, Wolfe CD. Natural history and effects on 2-year outcomes of urinary incontinence after stroke. Stroke; a journal of cerebral circulation 200 1;32(1):122-127.

5. Weatherall M, Slow T, Wiltshire K. Risk factors for entry into residential care after a support-needs assessment. The New Zealand medical journal 2004; 1 17(1202):U1075.

6. Füsgen I MH. nkontinenzmanual. Vol 55. Berlin-Heidelberg-New York: Springer; 1997

7. Langa KM, Fultz NH, Saint S, Kabeto MU, Herzog AR. Informal caregiving time and costs for urinary incontinence in older individuals in the United States. Journal of the American Geriatrics Society 2002;50(4):733-737.

8. Santini S, Andersson G, Lamura G. Impact of incontinence on the quality of life of caregivers of older persons with incontinence: A qualitative study in four European countries. Archives of gerontology and geriatrics 2016;63:92-101.

9. Markland AD, Vaughan CP, Johnson TM, 2nd, Burgio KL, Goode PS Incontinence. The Medical clinics of North America 201 1;95(3):539554, $x-x i$

10. DuBeau CE. Beyond the bladder: management of urinary incontinence in older women. Clinical obstetrics and gynecology 2007;50(3):720-734

11. Inouye SK, Studenski S, Tinetti ME, Kuchel GA. Geriatric syndromes: clinical, research, and policy implications of a core geriatric concept. Journal of the American Geriatrics Society 2007;55(5):780-791.

12. Resnick NM, Yalla SV, Laurino E. The pathophysiology of urinary 
incontinence among institutionalized elderly persons. The New England journal of medicine 1989;320(1):1-7

13. Taylor JA $3^{\text {rd }}$, Kuchel GA. Detrusor underactivity: Clinical features and pathogenesis of an underdiagnosed geriatric condition. Journal of the American Geriatrics Society 2006;54(12): 1920-1932.

14. Ouslander JG. Intractable incontinence in the elderly. BJU international 2000;85 Suppl 3:72-78; discussion 81-72

15. Mardon RE, Halim S, Pawlson LG, Haffer SC. Management of urinary incontinence in Medicare managed care beneficiaries: results from the 2004 Medicare Health Outcomes Survey. Archives of internal medicine 2006; 166(10): 1 128-1133.

16. Wagg A, Gibson W, Ostaszkiewicz J, et al. Urinary incontinence in frail elderly persons: Report from the 5th International Consultation on Incontinence. Neurourology and urodynamics 2015;34(5):398-406.

17. Holroyd-Leduc JM, Tannenbaum C, Thorpe KE, Straus SE. What type of urinary incontinence does this woman have? Jama 2008;299(12): 1446-1456

18. Christofi N, Hextall A. An evidence-based approach to lifestyle interventions in urogynaecology. Menopause international 2007; 13(4): 154-158.

\section{FRAGILITY FRACTURES AND REHABILITATION}

\section{Eleftheria Antoniadou}

Definition: A fragility fracture is a low energy fracture resulting from a simple fall from the standing height or less or from mechanical forces that would not ordinarily cause a fracture'.

Fragility Fracture Location ${ }^{2}$ : Potentially any bone falling within the above definition. The most common are hip, spine, near arm, carpal joint.

Pathophysiology: Three pathophysiological pathways are known for a fragility fracture. Low bone density (mass/ volume), as in osteoporosis.

Deterioration of the collagen structure as in Osteogenesis Imperfecta. Increased bone without subsequent remodeling such as Paget's Disease or Osteopetrosis.

Epidemiological data: The risk of a white woman's in her 50 s to present a hip fracture is $17 \%$. At the age of 90 , is 1 in 3. At the same time, she has a $15 \%$ chance of having a Colles' fracture and $32 \%$ of a vertebral fracture.

Generally, the incidence of fragility fractures over a woman's lifetime is $40-50 \%$, and that of a male $13-22 \%$. If we take into account the large demographic aging of the population, we can understand the huge impact of these disease entities on the population as well as on health systems.

The hip fracture shows $4 \%$ surgical mortality, higher in males and especially in the first 3 months. Mortality in the first postfracture year is about $20 \%$; this is usually because these fractures occur in the vulnerable population - the second loss that the elder's experience after a hip fracture is the decrease in function. In the geriatric frail population, this decrease is measured by the walking speed which is reduced to $40 \%$ on these patients. The third is the loss of autonomy, one out of three patients are admitted to a nursery home.
Vertebral fractures show similar mortality, and increased morbidity (pain, decreased respiratory function). After the $1^{\text {st }}$ vertebral fracture, there is a $20 \%$ chance that a second occurs ("fracture cascade"). The presence of a previous vertebral fracture increases the chance of disability and loss of autonomy in Activities of Daily Living (ADL) and, of course, affects the quality of life. The most common vertebral fractures levels are T6-8 and L12-S1. The severity of the initial deformity of the vertebra is a poor prognostic marker and is measured by the Spinal Deformity Index (SDI) ${ }^{3}$. The spinal deformity index (SDI) is a semi-quantitative measure of the vertebral fracture status, counting both the number and severity of vertebral fractures. For each vertebra, a visual grade of $0,1,2$, or 3 is assigned. No is 0 , mild fracture $(20-25 \%)$ is grade 1 , moderate $(26-40 \%)$ corresponds to 2 , and severe fracture (more than $41 \%$ ) is 3 . The SDI is calculated by summing the fracture grades of all vertebrae ${ }^{4}$. Let's not forget that the first fragility fracture will bring more 5 .

At the same time, the cost of hospitalization and rehabilitation of these fractures in Greece ${ }^{6}$ is as follows:

\begin{tabular}{|l|c|c|}
\hline Location & Cost $(€)$ & $95 \% \mathrm{Cl}(€)$ \\
\hline Hip & $4,334.27$ & $1,452.86-10,730.17$ \\
\hline Spine & $2,723.27$ & $1,470.39-7,839.55$ \\
\hline Colles & $1,731.35$ & $1,131.17-1,942.48$ \\
\hline
\end{tabular}

The total medical cost of fragile fractures in Greece is $€ 890,000,000$.

Diagnosis: The likelihood of fragility fracture is dependent on the presence or absence of osteoporosis and the risk of falling. Therefore, all clinical factors for primary and secondary osteoporosis as well as balance disorders are risk factors for a fragility fracture. For the diagnosis of osteoporosis, we use clinical and laboratory tools. Anamnesis to search osteoporotic fractures in the family of the patient, a medication used, diseases causing osteoporosis, but also receiving or nondairy and menopause age. Clinical examination-measurements like the distance between occiput and wall, height and weight, deformities in the spine, and in the countries where is available the FRAX. The FRAX is a computer-based algorithm of the 10-year likelihood for a major osteoporotic fracture created by the $\mathrm{WHO}^{7}$. For people aged up to 75 years, treatment starts at a rate of $2.5 \%$ for hip fracture and $10 \%$ for major osteoporotic fracture, while for people 75 years $5 \%$ and $15 \%$, respectively. If needed we proceed to DXA Scan as the gold standard method for assessing bone mass by measuring bone mineral density $(B M D)^{8}$. The patient's BMD is compared to the healthy individuals of the same ethnicity, age, and sex and gives us the Z-score, but also to that of the young adults and so we have the T-score that reflects the maximum bone mass. Conventional osteoporosis is defined by WHO as T-score (lumbar or hip) less than -2.5 (SD) ${ }^{8}$, and osteopenia from -1 to $-2.5 \mathrm{SD}$. Also when needed spine RX, necessary to 
investigate any vertebral fractures. The basic biochemical markers assessed are Ca, Vit D3, alkaline phosphatase, and blood) as a basic test and more complex bone markers as an important assessment of the bone turnover.

Regarding the assessment of the risk of falling in the geriatric population, the Comprehensive Geriatric Assessment with a focus on exploring balance disorders is an acceptable strategy. The interdisciplinary team evaluating the patient should determine which of the falls are considered pathological and then proceed to medical history with emphasis on possible causes of falls, a number of pharmaceutical preparations used and especially the ones acting on the CNS, last ophthalmological assessment, social history, appropriateness of the house. A very important part in the assessment is devoted to scales measuring function such as MNA (measuring nutritional adequacy), Berg, miniBEST, Tinetti (equilibrium estimation scales), SPPB (Risk of falling, and more general physical function), walking speed (Best Performance Index for the frail elder).

Significant measurements in this group are also the ones that estimate dementia such as MMSE, and depression, such as GDS. Scales that measure the ADL and IADL (instrumental $A D L)$ can be incorporated. Paramount is also to diagnose frailty using the following tools, Fried phenotype, the Frailty Index of accumulative deficits, and the Frailty Trait Scale ${ }^{8}$.

\section{Hip fracture and rehabilitation}

One of the most basic questions that should be answered in this patient group is what rehabilitation program is the ideal one. The systematic review of Chudyk tries to answer this question ${ }^{4}$.

This review was based on data from Oxford Center for Evidence-Based Medicine Levels of Evidence level of I (randomized controlled trial, RCT) or II (cohorts). The methodological quality of the studies was assessed with the modified version of the Downs and Black checklist. The composition of the data is as follows. 55 studies met the screening criteria: 30 RCTs and 25 nonRCTs. These studies were divided into 6 categories of interventions in rehabilitation (health pathways, early rehabilitation, interdisciplinary care, occupational therapy and physiotherapy, exercise, nonspecific intervention). The analysis concludes:

I. For the walking ability the best strategies in rehabilitation are:

- Postoperative management by a geriatrician with highfrequency occupational therapy and physiotherapy in the acute phase.

- Inpatient rehabilitation with occupational therapy and physiotherapy program along with treadmill training and body weight exercises as well as neuromuscular stimulation of the quadriceps.

- Home-based rehabilitation, a combination of aerobic exercise and resistance exercises combined with functional training.

II. In functional rehabilitation, the best strategies are:

- All the previous ones but adding quick mobilization and quick discharge with continued support.
III. In functional rehabilitation in patients with dementia (mild or moderate), there is no difference.

IV. Balance restoration:

- Occupational therapy and physiotherapy during hospitalization in a recovery structure together with quadriceps training and/or neuromuscular re-training of the same.

- In outpatient rehabilitation, physiotherapeutic intervention should be organized with a progressively increasing program based on aerobic exercise and resistance exercises.

Attention: A simple home physical therapy program has had the greatest risk of readmission!

The analysis in this review has had the following methodological problems:

- Large variation in the assessment of the effectiveness of the studies and their design.

- Absence of outcome measurements.

- Differentiation to endogenous program components.

- Evaluation of the results was incomplete, making it difficult to reproduce.

- Demographic variations of the intervention population and the control population.

\section{Vertebral fractures and rehabilitation}

The bibliography is poorer in terms of rehabilitation after a vertebral fracture. One interesting study on the subject is the study by Pratelli et al. ${ }^{2}$.

The treatment of vertebral fractures fragility can be conservative or surgical.

Conservative treatment is divided into:

- Pharmacological (Osteoporosis + Analgesic).

- Non-pharmacological (exercise, etc.). The rest of the conservative treatment is not adequately proven.

$1^{\text {st }}$ Stage of Conservative Therapy:

- Rest for 2 weeks or less and learn the right places on a bed.

- Exercises that do not move the spine.

- Upper and lower extremity exercises.

- Orthosis (TLSO) 8-12 weeks.

Stage 2 up to two months after:

- Avoid intensive strengthening.

- Relaxation techniques.

- Preserve range of motion.

- Breathing exercises.

$3^{\text {rd }}$ Stage 8-12 weeks with the removal of the orthosis (based on radiography and absence of pain):

- Strengthening exercises of the extensors.

- Posture training.

- Balance training.

- Proprioception exercises.

The exercises (stabilization, balance, and stretching) reduce chronic pain and disability. Time, a way of exercising and venue depends on the patient and his goals. Suggested session time is $45-50 \mathrm{~min}$. 


\section{Conclusions}

In conclusion, we must emphasize the enormous epidemiological, medical and financial burden of fragility fractures in a class of patients who are usually suffering from multimorbidity and frailty. Still, there are many questions, especially in rehabilitation, which should be answered by the scientific community.

\section{References}

1. Cummings SR, Melton LJ. Epidemiology and outcomes of osteoporotic fractures. The Lancet 2002; 359(9319):1761-1767.

2. Pratelli E, Cinotti I, Pasquetti P. Rehabilitation in osteoporotic vertebral fractures. Clin Cases Miner Bone Metab 2010;7(1):45-7.

3. AntoniadouE, Kouzelis A, Diamantakis G, BavelouA, Panagiotopoulos
E. Characteristics and diagnostic workup of the patient at risk to sustain fragility fracture. Injury 2017;48(Suppl 7):S17-S23.

4. Chudyk AM, Jutai JW, Petrella RJ, Speechley M. Systematic Review of Hip Fracture Rehabilitation Practices in the Elderly. Archives of Physical Medicine and Rehabilitation 2009;90(2):246-262.

5. Friedman SM, Mendelson DA. Epidemiology of Fragility Fractures. Clinics in Geriatric Medicine 20 14;30(2):175-181.

6. Athanasakis K, Karampli E, Ollandezos M, Papagiannopoulou V, Badamgarav E, Intorcia M, Kyriopoulos J. The Economic Burden of PostMenopausal Osteoporosis and Related Fractures in Greece. Value in Health 14(7).

7. WHO. Assessment of osteoporosis at the primary health care level. Geneva: WHO; 2007 [At: www.who.int/chp/topics/rheumatic/en/ index.html. Accessed May 2012.

8. Assessment of fracture risk and its application to screening for postmenopausal osteoporosis. Geneva, Switzerland: World Health Organization; 1994. 